\title{
Single-Cell Phosphospecific Flow Cytometric Analysis of Canine and Murine Adipose-Derived Stem Cells
}

\author{
Harumichi Itoh, ${ }^{1}$ Shimpei Nishikawa, ${ }^{1}$ Tomoya Haraguchi, ${ }^{1}$ Yu Arikawa, \\ Masato Hiyama, ${ }^{2}$ Toshie Iseri, ${ }^{3}$ Yoshiki Itoh, ${ }^{3}$ Munekazu Nakaichi, ${ }^{3}$ Yasuho Taura, ${ }^{2}$ \\ Kenji Tani, ${ }^{2}$ and Kazuhito Itamoto ${ }^{1}$ \\ ${ }^{1}$ Department of Small Animal Clinical Science, Joint Faculty of Veterinary Medicine, Yamaguchi University, 1677-1 Yoshida, \\ Yamaguchi City, Yamaguchi 753-8515, Japan \\ ${ }^{2}$ Department of Veterinary Surgery, Joint Faculty of Veterinary Medicine, Yamaguchi University, 1677-1 Yoshida, \\ Yamaguchi City, Yamaguchi 753-8515, Japan \\ ${ }^{3}$ Department of Veterinary Radiology, Joint Faculty of Veterinary Medicine, Yamaguchi University, 1677-1 Yoshida, \\ Yamaguchi City, Yamaguchi 753-8515, Japan \\ Correspondence should be addressed to Shimpei Nishikawa; sn2007@yamaguchi-u.ac.jp
}

Received 17 March 2017; Revised 28 May 2017; Accepted 6 June 2017; Published 16 August 2017

Academic Editor: Masanori Tohno

Copyright (c) 2017 Harumichi Itoh et al. This is an open access article distributed under the Creative Commons Attribution License, which permits unrestricted use, distribution, and reproduction in any medium, provided the original work is properly cited.

This study aimed to demonstrate single-cell phosphospecific flow cytometric analysis of canine and murine adipose-derived stem/stromal cells (ADSCs). ADSCs were obtained from clinically healthy laboratory beagles and C57BL/6 mice. Cell differentiation into adipocytes, osteocytes, and chondrocytes was observed for the cultured canine ADSCs (cADSCs) and murine ADSCs (mADSCs) to determine their multipotency. We also performed single-cell phosphospecific flow cytometric analysis related to cell differentiation and stemness. Cultured cADSCs and mADSCs exhibited the potential to differentiate into adipocytes, osteocytes, and chondrocytes. In addition, single-cell phosphospecific flow cytometric analysis revealed similar $\beta$-catenin and Akt phosphorylation between mADSCs and cADSCs. On the other hand, it showed the phosphorylation of different Stat proteins. It was determined that cADSCs and mADSCs show the potential to differentiate into adipocytes, osteocytes, and chondrocytes. Furthermore, a difference in protein phosphorylation between undifferentiated cADSCs and mADSCs was identified.

\section{Introduction}

Adipose-derived stem/stromal cells (ADSCs) reside in adipose tissues and are considered to be the progenitors of adipocytes. A recent study revealed that ADSCs can differentiate into adipocytes, osteocytes, and chondrocytes [1]. Mesenchymal stem cells (MSCs), such as ADSCs and bone marrow-derived stem/stromal cells (BMSCs), have many clinical advantages over embryonic stem cells (ESCs) and induced pluripotent stem cells (iPSCs). As ESCs are pluripotent stem cells derived from blastocysts, the ethical issues regarding their use are still unresolved. In contrast, iPSCs are generated by transduction of the four Yamanaka factors into differentiated somatic cells [2]. However, it was suggested that they are associated with the risk of malignant transformation [3]. MSCs, on the other hand, are easily obtained from autologous tissues, thus minimizing these problems associated with ESCs and iPSCs. Additionally, ADSCs can be obtained less invasively than BMSCs as they are present in subcutaneous tissues [4]. Considering this background, ADSCs are expected to be useful in human and veterinary regenerative medicine.

The expression of cluster of differentiation (CD) molecules in murine and human ADSC has been reported. Specifically, some surface antigen markers, such as CD34, CD44, and CD90, have been detected as ADSC markers [5]. However, how these surface antigen markers are functionally related to cell differentiation or maintenance of ADSC 
stemness is not well understood. In contrast, the intracellular protein phosphorylation directly reflects cell differentiation, proliferation, migration, adhesion, and so on. In murine ADSCs (mADSCs), some studies have reported on protein phosphorylation as a means of differentiating adipocytes, osteocytes, chondrocytes, and others. However, most of these reports describe comparisons between before and after the induction of differentiation. Furthermore, there has been no comprehensive study on protein phosphorylation with regard to ADSCs. In veterinary medicine, multipotency to differentiate into adipocytes, osteocytes, and chondrocytes has been identified in cADSCs. However, the details of differentiation mechanism have not been well identified.

In this study, we performed a comprehensive analysis of protein phosphorylation in mADSCs and cADSCs. We also compared protein phosphorylation between these two types of cell.

\section{Materials and Methods}

2.1. Ethical Approval. The institutional animal experiment ethics committee approved the animal experiments in this study. This work was conducted in accordance with the institutional guidelines of Yamaguchi University.

2.2. Animals and ADSC Isolation. Subcutaneous adipose tissue was collected from clinically healthy laboratory beagles (an 8-year-old female weighing $9.3 \mathrm{~kg}$ and a 6-year-old male weighing $9.0 \mathrm{~kg}$ ) and C57BL/6J mice purchased from a private company (Kyudo Co., Ltd., Saga, Japan). cADSCs and mADSCs were isolated as previously described [6]. To obtain adipose tissues from laboratory beagles, anesthesia was induced in the dogs using $7 \mathrm{mg} / \mathrm{kg}$ propofol (1\% propofol injection; Intervet, Tokyo, Japan) and maintained with isoflurane (Isoflurane, DS Pharma Animal Health Co., Osaka, Japan) in oxygen. In addition, $20 \mu \mathrm{g} / \mathrm{kg}$ buprenorphine (Lepetan ${ }^{\circledR}$ Otsuka Pharmaceutical, Tokyo, Japan) and $0.2 \mathrm{mg} / \mathrm{kg}$ meloxicam (Metacam ${ }^{\circledR}$ Boehringer Ingelheim, Tokyo, Japan) were used as analgesics. Adipose tissue was washed with Dulbecco's phosphate-buffered saline (DPBS; Wako, Osaka, Japan) and cut into fine pieces that were incubated at $37.5^{\circ} \mathrm{C}$ for $1 \mathrm{~h}$ with shaking in high-glucose Dulbecco's modified Eagle's medium (Wako) supplemented with $10 \%$ fetal bovine serum (FBS; Sigma-Aldrich, St. Louis, MO, USA), penicillin $(100 \mathrm{U} / \mathrm{mL}) /$ streptomycin $(100 \mu \mathrm{g} / \mathrm{mL})$, amphotericin B $(0.25 \mu \mathrm{g} / \mathrm{mL})(100 x$ Antibiotic-Antimycotic Mixed Stock Solution; Nacalai Tesque, Kyoto, Japan), and collagenase type I $(1.0 \mathrm{mg} / \mathrm{mL}$; Sigma-Aldrich). A 100- $\mu$ m nylon mesh (EASYstrainer, $100 \mu \mathrm{m}$; Greiner Bio-One Japan, Tokyo, Japan) was used to filter digested tissues, followed by centrifugation at $1,800 \mathrm{rpm}$ for $5 \mathrm{~min}$ in FACS buffer composed of $30 \mathrm{~mL}$ of DPBS supplemented with $1 \%$ FBS and $1 \mathrm{mM}$ EDTA.3Na (Wako). The pellet was seeded on culture plates and reached 80\%-90\% confluence; ADSCs were replated using trypsinEDTA (0.25\%).

2.3. Cell Differentiation and Immunofluorescence Staining. A cell differentiation kit (Mouse Mesenchymal Stem Cell Functional Identification Kit; R\&D Systems, Minneapolis,
MN, USA) was used to analyze the adipogenic, osteogenic, and chondrogenic differentiation potential of ADSCs, in accordance with the manufacturer's instructions.

To detect adipogenic differentiation by immunocytochemistry, cells were incubated for $1 \mathrm{~h}$ in DPBS containing $10 \mu \mathrm{g} / \mathrm{mL}$ goat anti-mouse fatty acid-binding protein (FABP) 4 antibody to label adipocytes. Cells were washed with DPBS and incubated for $1 \mathrm{~h}$ in DPBS containing phycoerythrin(PE-) conjugated secondary antibody [rabbit $\mathrm{F}\left(\mathrm{ab}^{\prime}\right) 2$ antigoat IgG H\&L (PE), preadsorbed; Abcam ${ }^{\circledR}$ Japan, Tokyo, Japan]. After washing with DPBS, cells were mounted with a solution containing $5 \mu \mathrm{g} / \mathrm{mL}$ Hoechst 33342 (Dojindo Laboratories, Kumamoto, Japan) to stain nuclei.

To detect osteogenic differentiation by immunocytochemistry, cells were then incubated for $1 \mathrm{~h}$ in DPBS containing $10 \mu \mathrm{g} / \mathrm{mL}$ goat anti-mouse osteopontin antibody to label osteocytes. After washing with DPBS, cells were incubated for $1 \mathrm{~h}$ in DPBS containing PE-conjugated rabbit anti-goat secondary antibody, washed with DPBS, and mounted as described above.

To detect chondrogenic differentiation by immunocytochemistry, the pellet was fixed and cut into sections with a thickness of 5-10 $\mu \mathrm{m}$ using a specimen matrix [Embedding Medium for Frozen Tissue Specimens to ensure Optimal Cutting Temperature (OCT); Sakura Finetek USA, Torrance, CA, USA] and a cryostat (CM 1950; Leica, Melbourne, Australia). After cutting into sections and placing on a slide, the slide was incubated for $1 \mathrm{~h}$ in DPBS containing $10 \mu \mathrm{g} / \mathrm{mL}$ sheep anti-mouse collagen II antibody to label chondrocytes. After washing with DPBS, cells were incubated for $1 \mathrm{~h}$ in DPBS containing PE-conjugated donkey anti-sheep secondary antibody [Donkey F( $\left.\mathrm{ab}^{\prime}\right) 2$ Anti-Sheep IgG H\&L (Phycoerythrin) preadsorbed; Abcam], washed with DPBS, and mounted as described above. For a negative control, we used DPBS without a primary antibody for all differentiation potentials.

2.4. Single-Cell Phosphospecific Flow Cytometric Analysis. Adherent cells from passage 4 were dissociated and cells were resuspended and incubated in FACS buffer containing $2 \mu \mathrm{L}$ of Fc receptor-blocking reagent (FcX Blocker; Biolegend, San Diego, CA, USA). Cells were stained with reagent to exclude dead cells (Zombie NIR; Tomy Digital Biology Co., Ltd., Tokyo, Japan). After washing the cells, IC fixation buffer (Affymetrix Japan, Tokyo, Japan) was added with shaking and then incubated. Permeabilization buffer (Affymetrix Japan) was diluted with purified water 10 times and added to wash the cells. After permeabilization, antibodies were used to stain each phosphorylated protein, as shown in Table 1. All antibodies were supplied by BD Biosciences (Tokyo, Japan). Stained cells were sorted by flow cytometry (Accuri C6; BD) and data were analyzed with FlowJo software (Tree Star, Ashland, OR, USA). Phosphorylation of proteins was considered to be positive when over $10 \%$ of all cells were stained, in contrast to the isotype control.

\section{Results}

3.1. Cell Differentiation into Adipocytes, Osteocytes, and Chondrocytes. Both mADSCs and cADSCs passaged four times 


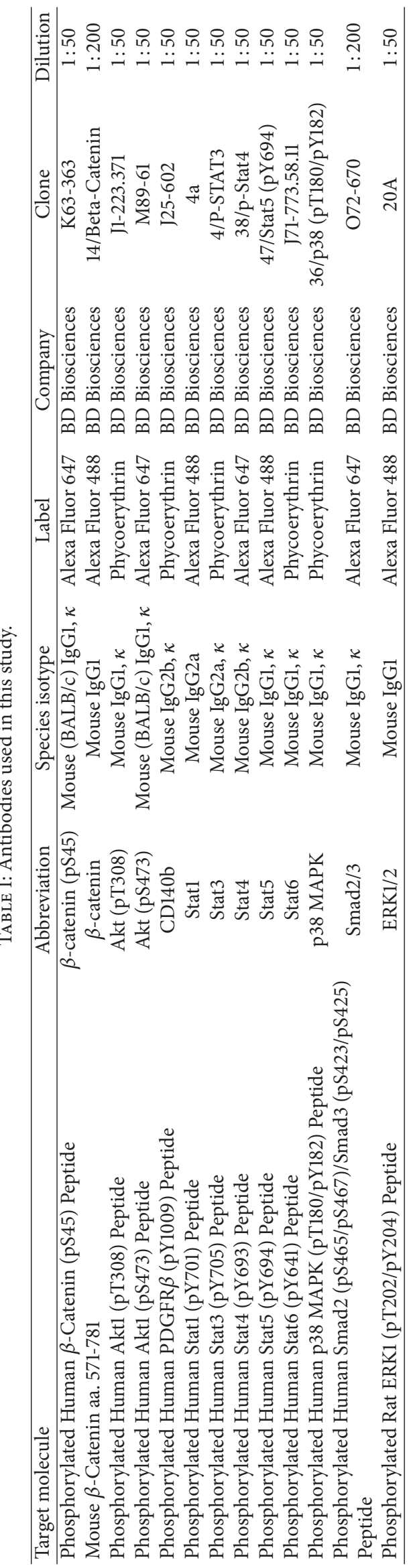


and displayed spindle-shaped morphology, as determined with phase-contrast microscopy (Figures $1(\mathrm{a})$ and 1(b)). To assess the adipogenic, osteogenic, and chondrogenic differentiation potentials of cells, cADSCs and mADSCs were differentiated into these lineages using in vitro differentiation to each subpopulation. After this in vitro differentiation, antiFABP4 immunofluorescence staining revealed that ADSCs that differentiated into adipocytes appeared as accumulated lipid droplets in the cytosol (Figures 1(c) and 1(d)). Antiosteopontin immunofluorescence staining revealed that ADSCs that differentiated into osteocytes appeared as accumulated granules in the cytosol (Figures 1(e) and 1(f)). Finally, anti-collagen II immunofluorescence staining revealed that ADSCs that differentiated into chondrocytes appeared as a fibrous formation in the cytosol (Figures $1(\mathrm{~g})$ and $1(\mathrm{~h})$ ).

3.2. Phosphorylation of Proteins. To clarify protein phosphorylation, $\beta$-catenin, $\beta$-catenin (pS45), Akt (pT308), Akt (pS473), CD140b, Stat1, Stat3, Stat4, Stat5, Stat6, p38 MAPK, Smad2/3, and ERK1/2 were measured using their respective antibodies. In mADSCs, $\beta$-catenin, $\beta$-catenin (pS45), Akt (pS473), Stat4, and Stat5 were detected, whereas Akt (pT308), CD140b, Stat1, Stat3, Stat6, p38 MAPK, Smad2/3, and ERK1/2 were not. Meanwhile, in cADSCs, $\beta$-catenin, $\beta$ catenin (pS45), Akt (pS473), and Stat4 were detected, and Akt (pT308), CD140b, Stat1, Stat3, Stat5, Stat6, p38 MAPK, Smad2/3, and ERK1/2 were not detected (Figure 2).

\section{Discussion}

In this study, we revealed that mADSCs and cADSCs have multipotency to differentiate into adipocytes, osteocytes, and chondrocytes. Both undifferentiated mADSCs and cADSCs expressed $\beta$-catenin, $\beta$-catenin pS45, Akt (pS473), and Stat 4 phosphorylation and only mADSCs expressed Stat5 phosphorylation.

Previous studies revealed that $\mathrm{WNT} / \beta$-catenin signaling inhibits adipogenesis in humans and mice. For example, the overexpression of Wntl or glycogen synthase kinase 3-b (GSK3b) phosphorylation-defective $\beta$-catenin mutant was shown to inhibit adipogenesis in 3T3-L1 preadipocytes (mouse fetal fibroblasts, which are widely used for metabolic study) by canonical pathway activation [7]. Another report revealed that pharmacological GSK3b inhibitors suppressed adipocyte differentiation [8].

However, to the best of our knowledge, there have been no previous reports on $\mathrm{WNT} / \beta$-catenin signaling and ADSC adipogenesis in canines. In this study, we also revealed $\beta$ catenin and $\beta$-catenin pS45 phosphorylation in MADSCs and cADSCs. This suggests that cADSCs also inhibit adipogenesis with $\mathrm{WNT} / \beta$-catenin signaling.

Akt, also known as protein kinase B, is a serine/threoninespecific protein kinase that plays roles in glucose metabolism, apoptosis, cell proliferation, transcription, and cell migration, among others [9]. In this study, we detected Akt pS473 phosphorylation in both mADSCs and cADSCs. Previous studies reported that Akt pS473 is phosphorylated by the mTOR-rictor pathway $[10,11]$. We identified a previous report on Akt expression in cADSCs; however, it did not describe the phosphorylation site of Akt [12]. The relationship between ADSCs and Akt has been reported in mADSCs. A previous study showed the accumulation of intracytoplasmic lipids with Akt phosphorylation in ADSCs with the inhibition of ErbB2 activity [13]. In murine 3T3-L1 preadipocytes, the inhibition of adipocyte differentiation was also found when Akt was inhibited [14]. Akt phosphorylation is also necessary for the differentiation of brown adipocytes, and PI3K inhibition blocks adipocyte differentiation in mice [15].

In this study, we detected the phosphorylation of Stat 4 and Stat5 in mADSCs, but only that of Stat 4 in cADSCs. A previous study revealed Stat 4 expression at the mRNA level in mADSCs [16]. However, we found no reports on Stat 4 expression at the protein level. Therefore, we concluded that this is the first report on Stat 4 expression in mADSCs and cADSCs.

Many clinical trials have been conducted for the ADSCrelated treatment of cardiovascular disease, spinal cord injury, cirrhosis, renal insufficiency, and skin fistula with Crohn's disease and for breast reconstruction after mastectomy in humans [17]. In veterinary medicine, we can also find some clinical trials for the ADSC-related treatment of acute thoracolumbar intervertebral disc disease [18], inflammatory bowel disease [19], chronic osteoarthritis [20], and so on. However, there has been insufficient basic research on cADSCs in veterinary medicine and the clinical trials that have been conducted provided limited evidence. In previous studies, comprehensive information on surface antigen markers or gene expression was reported for human ADSCs and mADSCs [5, 21, 22]. However, no comprehensive study on phosphorylation has been performed for a long time. In this study, some phosphorylated proteins were found to be expressed in undifferentiated mADSCs and cADSCs. However, it has not been revealed which signal pathways are expressed to maintain stemness in undifferentiated mADSCs and cADSCs. Therefore, it is necessary to investigate which signal is important to maintain stemness. Nonetheless, the present study provides a useful foundation for future research in veterinary medical science.

\section{Conclusion}

mADSCs and cADSCs showed adipogenic, osteogenic, and chondrogenic differentiation potential. mADSCs and cADSCs induced similar phosphorylation of $\beta$-catenin and Akt, in contrast to that of the Stat family.

\section{Conflicts of Interest}

The authors declare that there are no conflicts of interest regarding the publication of this paper.

\section{Acknowledgments}

This work was partly supported by a Japan Society for the Promotion of Science KAKENHI Grant (no. 26893172). The authors would like to thank staff of Yamaguchi University Animal Medical Center for their support. 


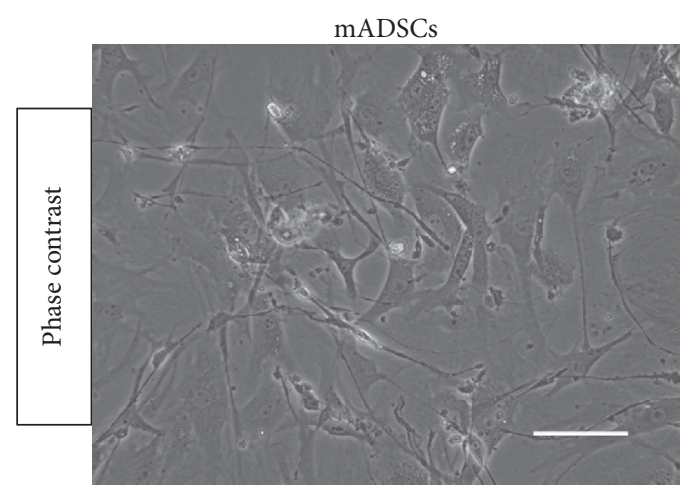

(a)

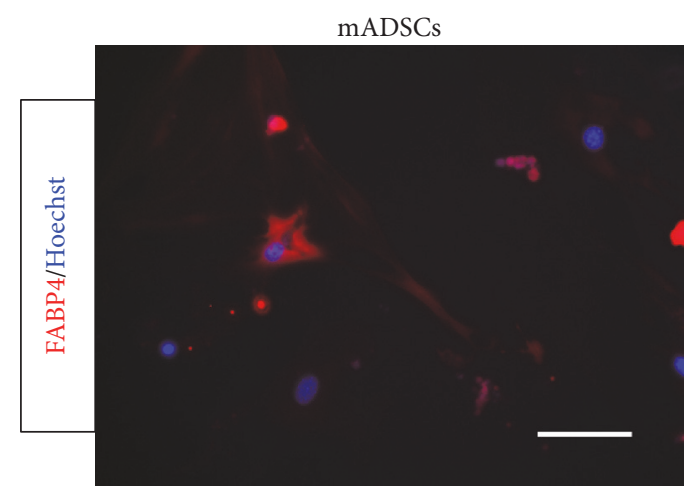

(c)

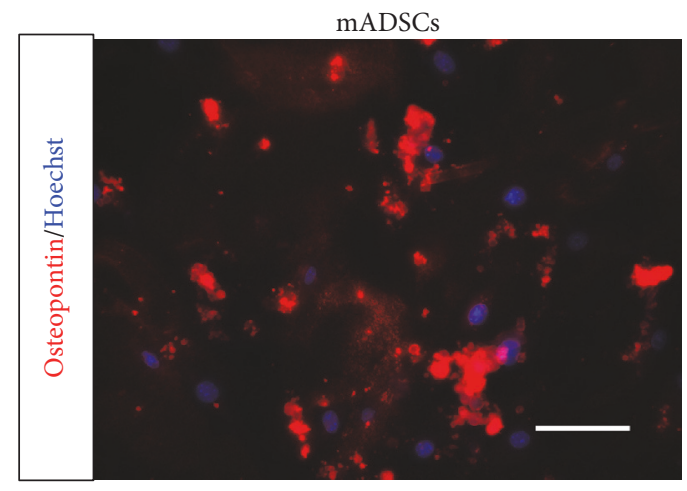

(e)

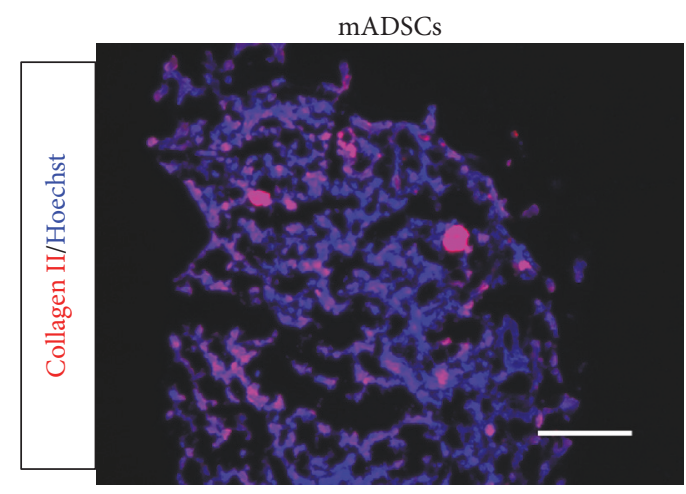

(g)

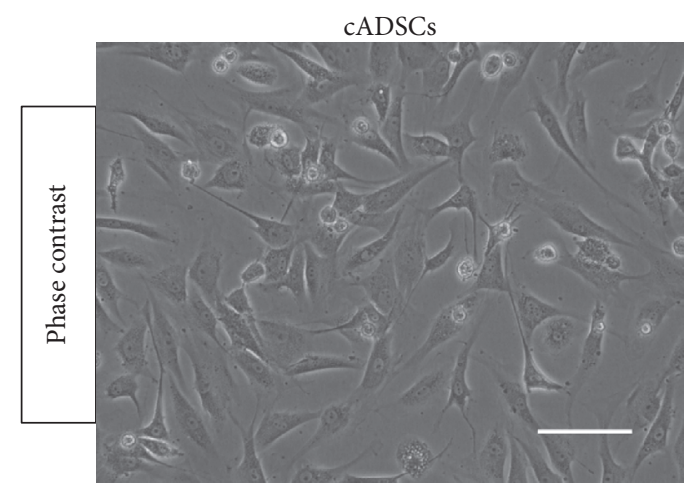

(b)

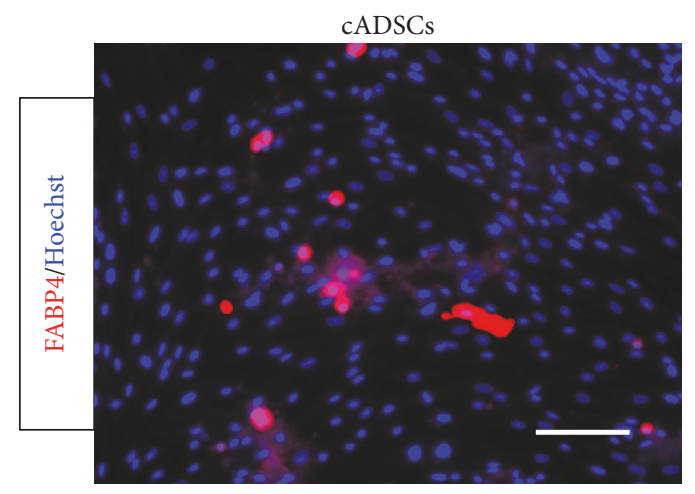

(d)

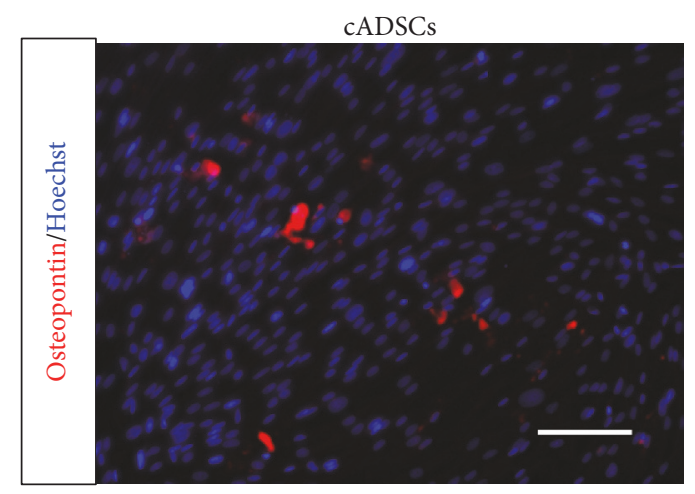

(f)

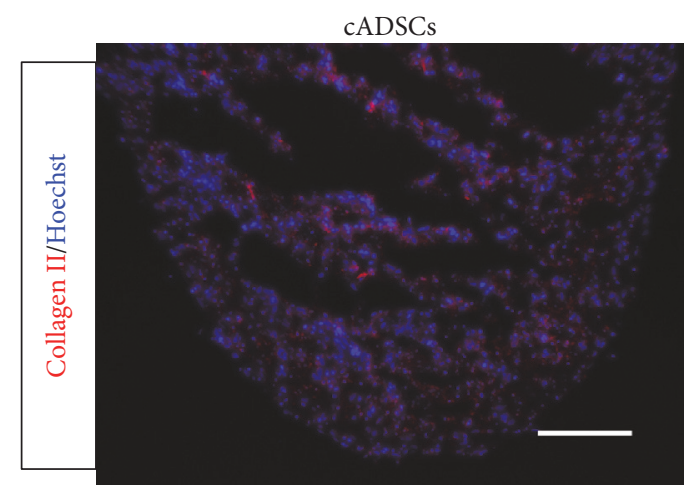

(h)

Figure 1: Differentiation potential of mADSCs (a, c, e, g) and cADSCs (b, d, f, h). Spindle-shaped morphology was identified by phasecontrast microscopy $(\mathrm{a}, \mathrm{b})$. FABP4 (c, d), osteopontin (e, f), and collagen II expression (red) following adipogenic (c, d), osteogenic (e, f), and chondrogenic $(\mathrm{g}, \mathrm{h})$ differentiation for mADSCs and cADSCs, as determined by immunocytochemistry. The nuclei were stained with Hoechst 33342 (blue). 

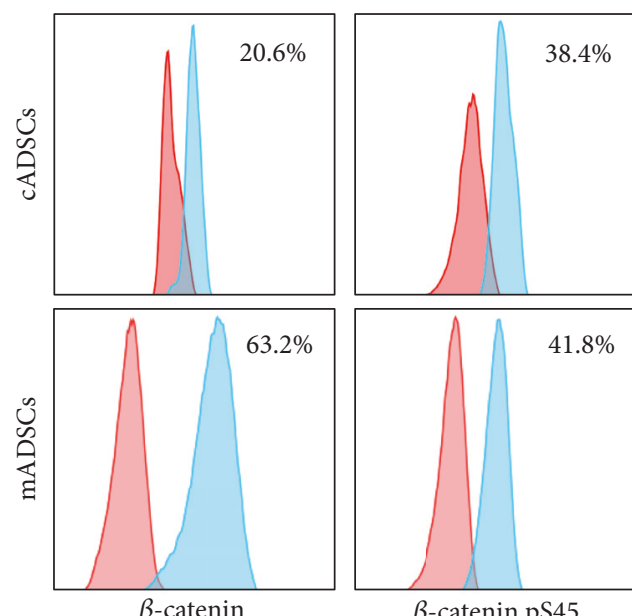

$\beta$-catenin pS45
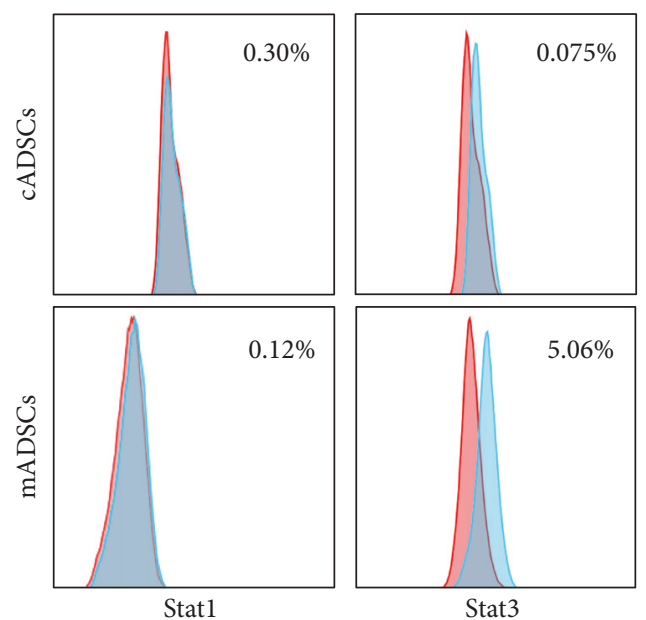

Stat3
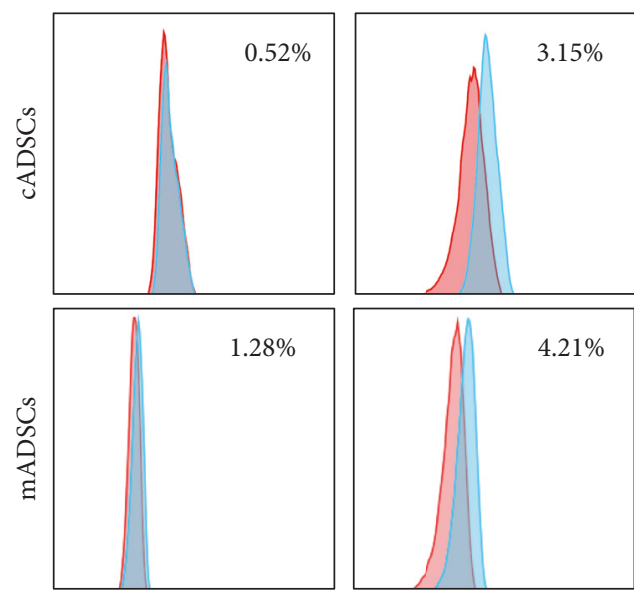

p38 MAPK

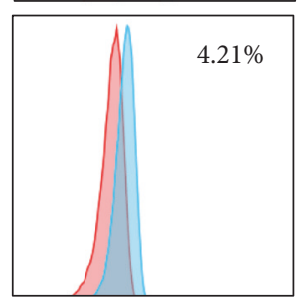

$\operatorname{Smad} 2 / 3$
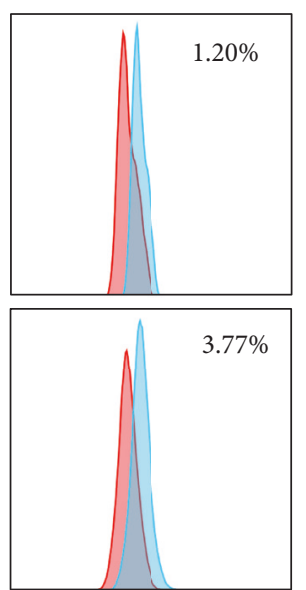

Akt (pT308)
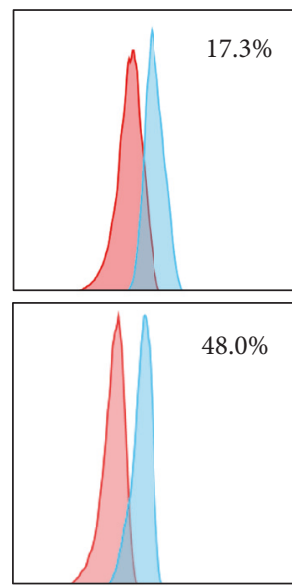

Stat 4
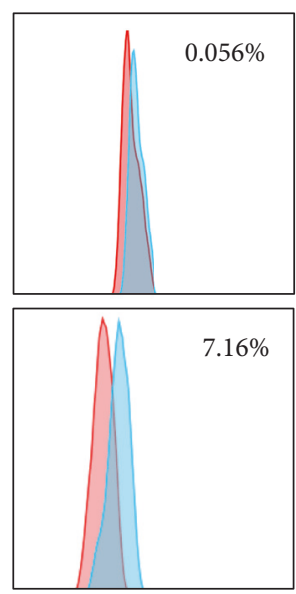

ERK1/2
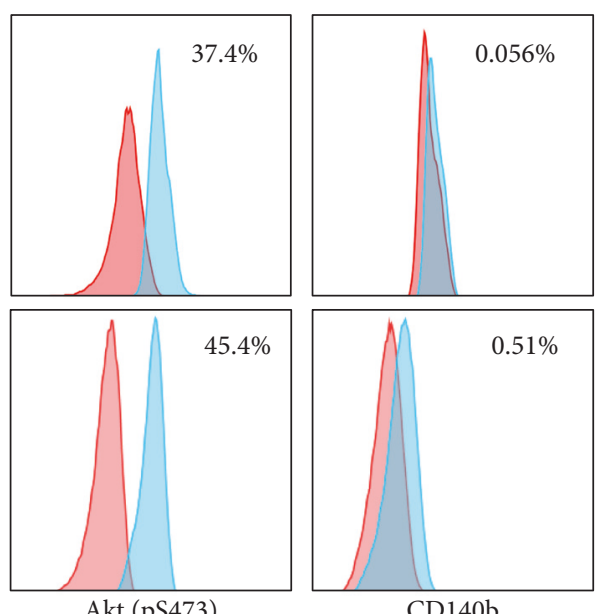

Akt (pS473)
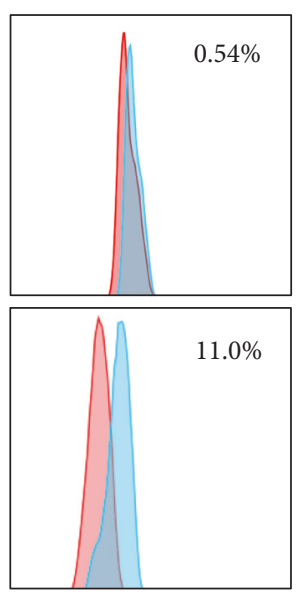

Stat5

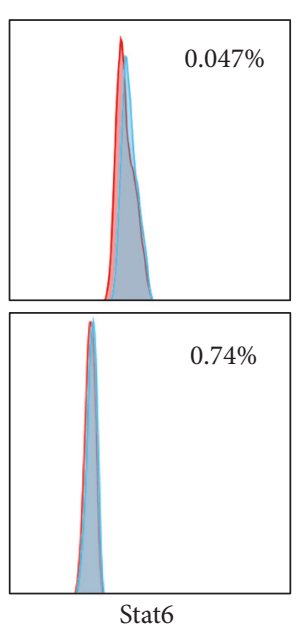

Stat6

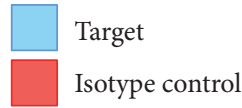

FIGURE 2: Comparison of protein phosphorylation of mADSCs and cADSCs. The red peaks represent the isotype controls, and the blue peaks represent antigens. $\beta$-catenin, $\beta$-catenin (pS45), Akt (pS473), Stat3, Stat4, Stat5, and ERK1/2 were detected, whereas Akt (pT308), CD140b, Stat1, Stat6, p38 MAPK, and Smad2/3 were not detected in mADSCs. In addition, $\beta$-catenin, $\beta$-catenin (pS45), Akt (pS473), and Stat4 were detected, whereas Akt (pT308), CD140b, Stat1, Stat3, Stat5, Stat6, p38 MAPK, Smad2/3, and ERK1/2 were negative in cADSCs. The percentages shown in the figure represent the positive rates of the antigen. 


\section{References}

[1] P. A. Zuk, M. Zhu, H. Mizuno et al., "Multilineage cells from human adipose tissue: implications for cell-based therapies," Tissue Engineering, vol. 7, no. 2, pp. 211-228, 2001.

[2] K. Takahashi and S. Yamanaka, "Induction of pluripotent stem cells from mouse embryonic and adult fibroblast cultures by defined factors," Cell, vol. 126, no. 4, pp. 663-676, 2006.

[3] K. Okita, T. Ichisaka, and S. Yamanaka, "Generation of germline-competent induced pluripotent stem cells," Nature, vol. 448, no. 7151, pp. 313-317, 2007.

[4] Y. Zhu, T. Liu, K. Song, X. Fan, X. Ma, and Z. Cui, "Adiposederived stem cell: a better stem cell than BMSC", Cell Biochemistry and Function, vol. 26, no. 6, pp. 664-675, 2008.

[5] Y. Kuroda and M. Dezawa, "Mesenchymal stem cells and their subpopulation, pluripotent muse cells, in basic research and regenerative medicine," Anatomical Record, vol. 297, no. 1, pp. 98-110, 2014.

[6] Y. Miyahara, N. Nagaya, M. Kataoka et al., "Monolayered mesenchymal stem cells repair scarred myocardium after myocardial infarction," Nature Medicine, vol. 12, no. 4, pp. 459-465, 2006.

[7] S. E. Ross, N. Hemati, K. A. Longo et al., "Inhibition of adipogenesis by Wnt signaling," Science, vol. 289, no. 5481, pp. 950953, 2000.

[8] C. N. Bennett, S. E. Ross, K. A. Longo et al., "Regulation of Wnt signaling during adipogenesis," The Journal of Biological Chemistry, vol. 277, no. 34, pp. 30998-31004, 2002.

[9] B. D. Manning and L. C. Cantley, "AKT/PKB signaling: navigating downstream," Cell, vol. 129, no. 7, pp. 1261-1274, 2007.

[10] G. P. Sapkota, L. Cummings, F. S. Newell et al., "BI-D1870 is a specific inhibitor of the p90 RSK (ribosomal S6 kinase) isoforms in vitro and in vivo," Biochemical Journal, vol. 401, no. 1, pp. 2938, 2007.

[11] M. Nakayama, T. Inoue, M. Naito, K. Nakayama, and N. Ohara, "Attenuation of the phosphatidylinositol 3-Kinase/Akt signaling pathway by Porphyromonas gingivalis gingipains RgpA, RgpB, and Kgp," Journal of Biological Chemistry, vol. 290, no. 8, pp. 5190-5202, 2015.

[12] S.-S. Park, Y. J. Lee, H. J. Han, and O.-K. Kweon, "Role of laminin-111 in neurotrophin-3 production of canine adiposederived stem cells: Involvement of Akt, mTOR, and p70S6K," Journal of Cellular Physiology, vol. 226, no. 12, pp. 3251-3260, 2011.

[13] C. Valerio, M. G. Scioli, P. Gentile et al., "Platelet-rich plasma greatly potentiates insulin-induced adipogenic differentiation of human adipose-derived stem cells through a serine/threonine kinase Akt-dependent mechanism and promotes clinical fat graft maintenance," Stem Cells Translational Medicine, vol. 1, no. 3, pp. 206-220, 2012.

[14] H. H. Zhang, J. Huang, K. Düvel et al., "Insulin stimulates adipogenesis through the Akt-TSC2-mTORC1 pathway," PLoS ONE, vol. 4, no. 7, Article ID e6189, 2009.

[15] M. Fasshauer, J. Klein, K. M. Kriauciunas, K. Ueki, M. Benito, and C. R. Kahn, "Essential role of insulin receptor substrate 1 in differentiation of brown adipocytes," Molecular and Cellular Biology, vol. 21, no. 1, pp. 319-329, 2001.

[16] K. Asano, S. Yoshimura, and A. Nakane, "Adipose tissue-derived mesenchymal stem cells attenuate staphylococcal enterotoxin A-induced toxic shock," Infection and Immunity, vol. 83, no. 9, pp. 3490-3496, 2015.
[17] M. Locke, V. Feisst, and P. R. Dunbar, "Concise review: human adipose-derived stem cells: separating promise from clinical need," Stem Cells, vol. 29, no. 3, pp. 404-411, 2011.

[18] Y. Kim, S. H. Lee, W. H. Kim, and O.-K. Kweon, “Transplantation of adipose derived mesenchymal stem cells for acute thoracolumbar disc disease with no deep pain perception in dogs," Journal of Veterinary Science, vol. 17, no. 1, pp. 123-126, 2016.

[19] E. M. Pérez-Merino, J. M. Usón-Casaús, C. Zaragoza-Bayle et al., "Safety and efficacy of allogeneic adipose tissue-derived mesenchymal stem cells for treatment of dogs with inflammatory bowel disease: clinical and laboratory outcomes," Veterinary Journal, vol. 206, no. 3, pp. 385-390, 2015.

[20] L. L. Black, J. Gaynor, D. Gahring et al., "Effect of adiposederived mesenchymal stem and regenerative cells on lameness in dogs with chronic osteoarthritis of the coxofemoral joints: a randomized, double-blinded, multicenter, controlled trial," Veterinary Therapeutics, vol. 8, no. 4, pp. 272-284, 2007.

[21] P. C. Baer, S. Kuçi, M. Krause et al., "Comprehensive phenotypic characterization of human adipose-derived stromal/stem cells and their subsets by a high throughput technology," Stem Cells and Development, vol. 22, no. 2, pp. 330-339, 2013.

[22] W. Wagner, F. Wein, A. Seckinger et al., "Comparative characteristics of mesenchymal stem cells from human bone marrow, adipose tissue, and umbilical cord blood," Experimental Hematology, vol. 33, no. 11, pp. 1402-1416, 2005. 

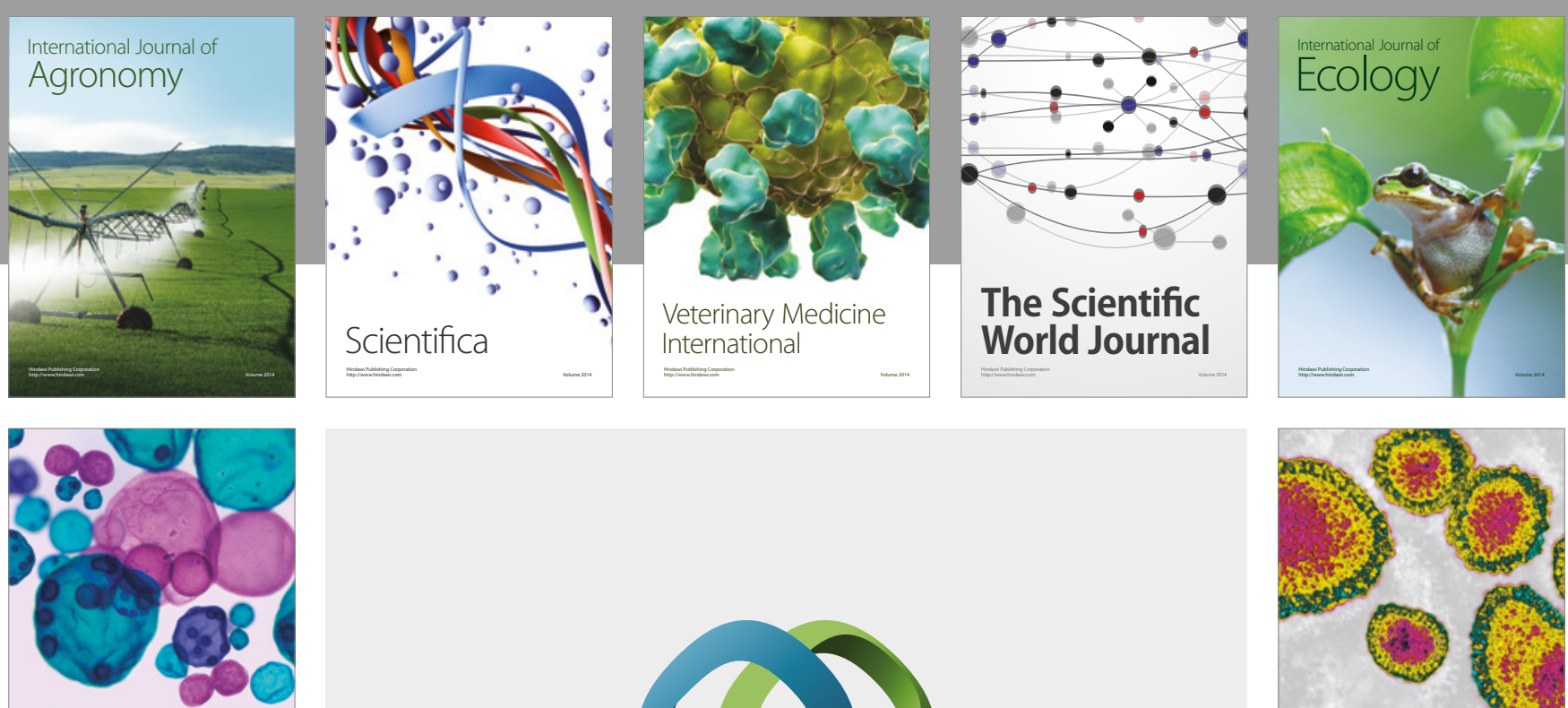

\section{International Journal of} Microbiology
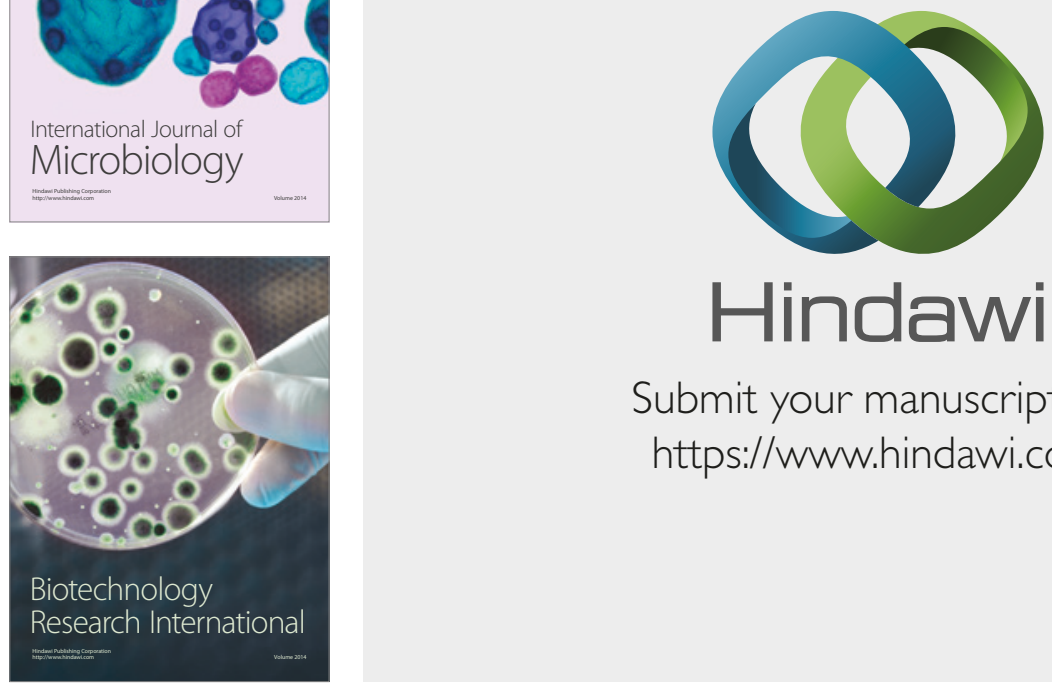

Submit your manuscripts at

https://www.hindawi.com
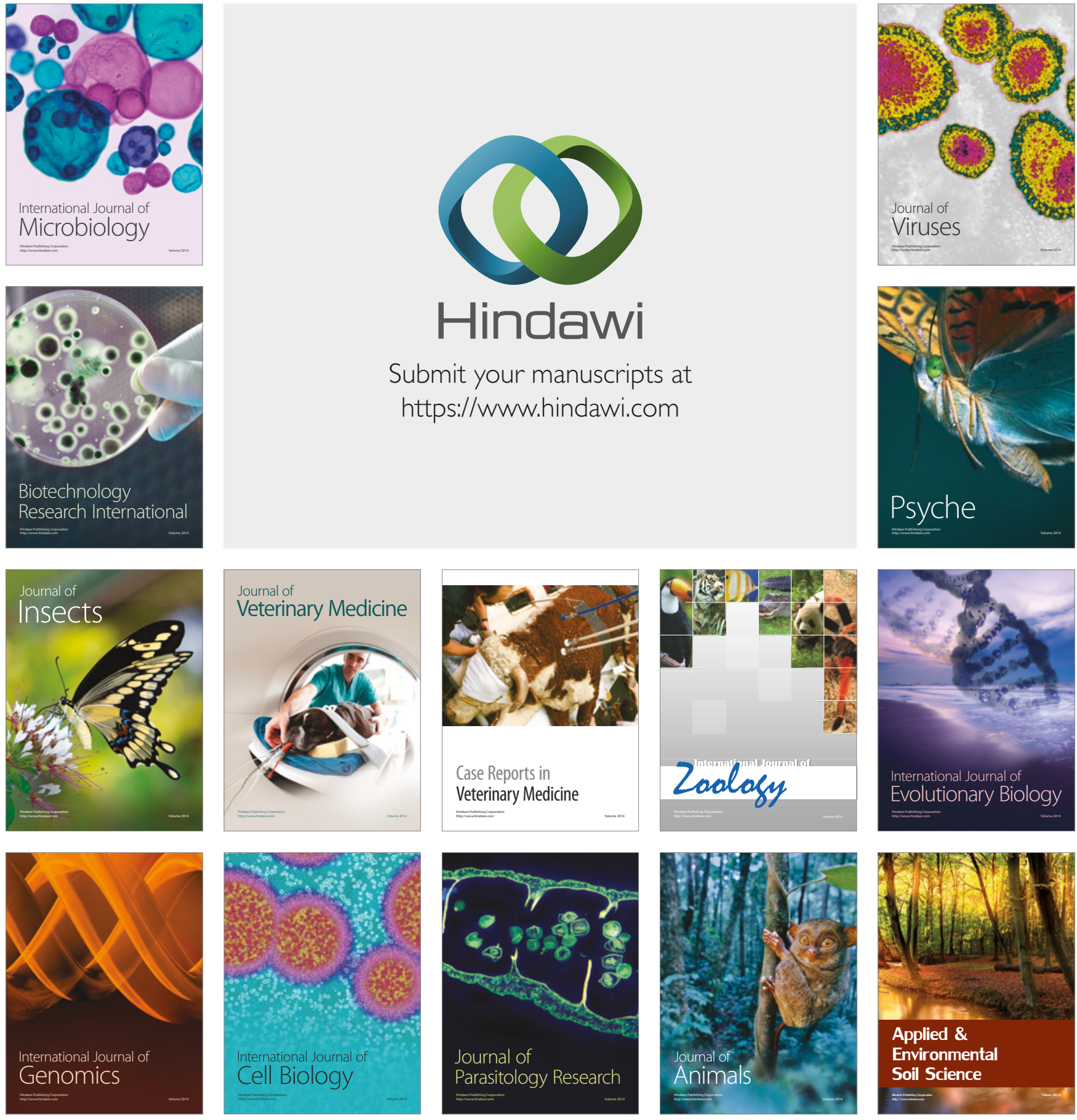